\title{
Differences between random-potential and random-magnetic-field localization in quasi-one-dimensional systems
}

\author{
W. L. Chan and X. R. Wang \\ Physics Department, The Hong Kong University of Science and Technology, Clear Water Bay, Hong Kong \\ X. C. Xie \\ Physics Department, The Hong Kong University of Science and Technology, Clear Water Bay, Hong Kong \\ and Physics Department, Oklahoma State University, Stillwater, Oklahoma 74078
}

(Received 7 December 1995; revised manuscript received 20 June 1996)

\begin{abstract}
We study electron localization of quasi-one-dimensional random-potential and random-magnetic-field systems with and without a uniform external magnetic field. We find that in a random-magnetic-field (RMF) system the localization length is not a monotonic decreasing function of magnetic-field randomness $\delta$ in contrast to a random-potential system in which the localization length is a monotonic decreasing function of the randomness $w$. We observed that, in both random-potential and RMF systems, the localization length can both increase and decrease with a uniform external magnetic field depending on the energy of a state. The crossover occurs when the electron energy is close to the band edge in a random-potential system. In a RMF system, the magnetoproperty of the localization length is complicated. A band edge effect is proposed to explain the anomalous numerical results. We also find that the inverse of the localization length of a quasione-dimensional disordered system peaks at certain energies inside the energy band. These peaks can be understood by the branch edge effect. [S0163-1829(96)09039-X]
\end{abstract}

\section{INTRODUCTION}

Recently there has been considerable interest in studying the problem of noninteracting two-dimensional electrons propagating in the presence of random magnetic flux. ${ }^{1-7}$ First, this problem arises in the study of the quantum Hall systems near even denominator filling fractions. ${ }^{1,8}$ Second, the problem is believed to be relevant to the theoretical studies of the high- $T_{c}$ models where gauge field fluctuations play an important role. ${ }^{9}$ Third, this problem has a pure academic interest of its own. It is well known that a usual randompotential system with the time-reversal symmetry has the coherent backward scattering which results in the weak localization effect. A system with random magnetic flux disorder does not have the time-reversal symmetry; nevertheless, localized states also exist. ${ }^{1-7,10}$ One of the interesting questions is to study differences of the localization properties of these two systems. We now know that the mobility edges can only exist in a space above two dimensions for a randompotential system, provided that disorders do not break the time-reversal symmetry. Another essential and controversial issue of the random magnetic flux problem (RMF) is the existence of mobility edges in a two-dimensional space.

A number of numerical investigations have been performed with conflicting results on the random field problem in a two-dimensional (2D) system. Pryor and Zee ${ }^{11}$ were the first to study the tight-binding model with the nearestneighbor and the next-nearest-neighbor interactions in a random magnetic field by direct diagonalization. They showed that in the presence of a strong random magnetic field in two dimensions most of the states are extended and only those states near the band edge are localized. Sugiyama and Nagaosa ${ }^{4}$ studied the same problem on a rectangular (strip) sample of size $L \times M$ with a periodic boundary condition by using the relation

$$
\xi^{-1}(L, E)=-\lim _{L \rightarrow \infty} \frac{1}{2 L} \ln \sum_{i, j}^{M}\left|G_{1 i, L j}(E)\right|^{2}
$$

where $\xi$ is the localization length of a state with energy $E$, $G_{1 i, L j}$ is the Green's function which connects two end points $(1, i)$ and $(L, j)$ of the long strip. They used MacKinnon's method $^{12}$ to calculate the Green's function on the lattice. The result is that all states are localized and as the electron energy approaches band center, the localization length increases rapidly. Kalmeyer et al. ${ }^{3}$ used both MacKinnon's method and the Landauer formula to study the same problem with hard-wall boundary conditions. Their studies suggest that there exist mobility edges in two dimensions when flux disorder breaks the time-reversal symmetry. Avishai et al. ${ }^{5}$ also used Landauer formula to study the same problem numerically. Their results are also consistent with the existence of extended states, and hence mobility edges in two dimensions. Liu et al. ${ }^{7}$ calculated localization length using the finite-size scaling method and also concluded that there is a metal-insulator transition in two dimensions. Recently, Avishai and Bar-Touv ${ }^{10}$ studied the interplay between potential and magnetic disorder in a double-chain ladder.

In this paper, we study a simple problem that noninteracting electrons propagate in a random potential and/or a random magnetic field in a quasi-one-dimensional lattice. Our focus is on the differences between the localization due to a random potential and that due to a random magnetic field in quasi-one-dimensional systems. In particular, we study the magnetoresponses and the phase randomness dependence of the localization length. It is believed that the localization 
length will increase in a weak magnetic field in the usual Anderson localization model because the field will destroy the time-reversal symmetry, thus it suppresses the coherent backward scattering. A magnetic field has, however, other effects on a wave function, such as changing phases of forward scatterings. To our knowledge, there is no detailed analysis of the energy dependence of the localization length in a weak magnetic field even for the Anderson localization model. Therefore, it will also be interesting to see to what extent one can say that the localization length will increase in a weak magnetic field. One of our results of the Anderson model is that the localization length of a state increases with a weak magnetic field if the state is near the band center. However, the localization length can also decrease when the state is close to band edge. This paper is organized as follows: In the next section, we will describe the model, its transfer matrix formulation, and the numerical method used to calculate the localization length (through Lyapunov exponents of a product of random matrices). The numerical results and their discussions are given in Sec. III.

\section{MODEL AND THEORETICAL METHOD}

\section{A. Model}

We consider a tight-binding model on a strip of $L \times M$

$$
\begin{aligned}
\mathcal{H}= & \sum_{i} \sum_{m=1}^{M} \varepsilon_{i m}|i m\rangle\langle i m| \\
& +\sum_{\langle i m ; j n\rangle}\left[t_{i m ; j n}|i m\rangle\left\langle j n\left|+t_{i m ; j n}^{\dagger}\right| j n\right\rangle\langle i m|\right],
\end{aligned}
$$

where $\langle i m ; j n\rangle$ indicates nearest neighbors on the lattice. The model is used to describe a noninteracting electron system in a random potential and/or random magnetic field with and without an external uniform magnetic field. $\varepsilon_{i m}$ is the on-site energy and is assumed to be uniformly distributed in $[-w / 2, w / 2]$. A magnetic field is introduced through the "Peierls ansatz,"13 namely, the only effect of a magnetic field on a tight-binding model is to add a phase to the hopping coefficient. We will choose a gauge in such a way that all phases are only acquired in the horizontal bonds, i.e., $t_{i m, i+1 m}=\eta e^{i \phi_{i, m} / 2}$ and $t_{i m, i m+1}=t$ for the intrachain and the interchain hopping coefficients, respectively. $\phi_{i, m}$ is assumed to be uniformly distributed in $[\phi-\delta \pi, \phi+\delta \pi]$, where $\phi$ corresponds to the uniform magnetic field. In this model, we have two disorder parameters $w$ and $\delta$. $w$ is the usual Anderson randomness, and $\delta$ is the phase randomness. The amplitude of the intrachain hopping coefficient is chosen as the unit of the energy $\eta=1$. It is interesting to point out that a magnetic field can always be gauged out in a one-chain system. In order to study the magnetic-field effects, the simplest quasi-one-dimensional system is the one with two chains, $M=2$. For a specific energy $E$, a transfer matrix $T_{i}$ which maps the wave-function amplitudes at column $i-1$ and $i$ to those at column $i+1$ can be easily set up. The asymptotic behavior of the wave function $\psi$ can be derived from the limit of the product of the transfer matrices

$$
P_{L} \equiv \prod_{i=1}^{L} T(i)
$$

It is known that Eq. (3) has a set of $M$ pairs of Lyapunov exponents, $^{2}\left(\gamma_{i},-\gamma_{i}\right)\{i=1,2, \ldots, M\}$, which can be related to physical quantities. The inverse of the localization length is given by the smallest positive Lyapunov exponent ${ }^{2}$ of the product of the random matrices $T(i)$. As $M$ increases from 1 to $\infty$, the system changes from a one-dimensional to a two-dimensional system. Therefore, the multichain system can also be used to investigate how the localization property of noninteracting particles changes from a $1 \mathrm{D}$ to a $2 \mathrm{D}$ system.

The energy of a pure double-chain system in the present of a uniform magnetic field can be solved exactly and is given by

$$
E=\cos (k a+\phi)+\cos (k a) \pm \sqrt{[\cos (k a+\phi)-\cos (k a)]^{2}+1}
$$

The above expression shows that the energy band shrinks with the magnetic field $\phi$. This effect will be important to understand how the magnetoproperties of the localization length depend on the energy.

\section{B. Numerical method}

As we have mentioned, the localization length is related to the smallest positive Lyapunov exponent of Eq. (3). However, if the product of random matrices $T(l)$ is computed directly, the information associated with the smallest positive Lyapunov exponent is lost when the ratio of the contribution from the smallest positive Lyapunov exponent to that of the largest Lyapunov exponent becomes comparable with the machine accuracy. ${ }^{12}$ In order to compute all Lyapunov exponents of the product of $T(l)$, we employ the following technique. ${ }^{12,14,15}$

Denote $B_{n}$ as the $n$th column of the product $P(l)$ of the transfer matrices $T(i)$. We perform the following GramSchmidt orthonormalization transformation on $B_{n}$ :

$$
\bar{B}_{n}=\left(B_{n}-\sum_{i<n}\left(B_{i} \cdot B_{n}\right) B_{i}\right) / b^{(n)},
$$

where

$$
b^{(n)}=\left|B_{n}-\sum_{i<n}\left(B_{i} \cdot B_{n}\right) B_{i}\right| .
$$

This transformation has to be performed regularly but not necessarily at each step. After the transformation, each column of the transfer matrix is orthogonal to the other. The first column converges to the eigenvector corresponding to the maximum eigenvalue. The second column converges to the eigenvector corresponding to the second maximum eigenvalue and so forth. Since the system is symplectic, the eigenvalues occur in reciprocal pairs. The logarithm of these eigenvalues are the Lyapunov exponents, thus the Lyapunov exponents of the product of $T(l)$ appear in pairs $\left(\gamma_{i},-\gamma_{i}\right)$ $\{i=1,2, \ldots, M\}$.

From the transformation equations (5) and (6), it is not difficult to recognize that $b^{(n)}$ is a measure of divergence of the norm of the column vector, resulting in the Lyapunov exponents. Therefore, for a system with $M$-chain system with length $L$, the localization length is given by 


$$
\xi^{-1}=\frac{c_{L}^{(M)}}{L}
$$

where

$$
c_{L}^{(M)}=\ln b_{L}^{(M)}+c_{L-1}^{(M)} .
$$

In our numerical calculations, all the $c_{0}^{(i)}$ 's are initialized to zero. Other Lyapunov exponents can be found by computing $b_{L}^{(i)}$ 's, and $c_{L}^{(i)}$ 's. Since the Lyapunov exponents of a product of random matrices are self-averaging quantities, the average procedure is granted for large $L$ systems. In general, if the matrix elements diverge very fast, one needs to reduce the number of steps between the sequential transformations. In the case of the localization problem we are studying, the optimal steps can be found easily in the following way. Since the random matrices are symplectic, the Lyapunov exponents appear in pairs. If the number of transformation is inadequate, no paired exponents can be obtained.

The above numerical technique is applied to both randompotential and random flux problems with the hard wall boundary conditions (HBC). The localization length is calculated with and without a uniform external magnetic field. The random matrices are generated by assigning a random number in the corresponding matrix element in $T(l)$. In our numerical calculation, we perform the transformation procedure for every 10 steps. The length $L$ of double-chain and triple-chain systems is $10^{6}$ and the length $L$ of a 20-chain system is $10^{5}$. The numerical error is about $10^{-4}$ for the double-chain and triple-chain calculations. The detailed numerical results are as follows.

\section{NUMERICAL RESULTS AND DISCUSSIONS}

\section{A. Peaks of the localization length at the branch edges}

We study the energy dependence of the localization length for both random-potential and random magnetic flux systems. The two curves with $\phi=0.0$ in Fig. 1 are the plots of inverse of the localization length versus energy in the absence of an external magnetic field, $w=1.0, \delta=0$, and $t=1$ is the curve for the random-potential problem, and $w=0, \delta=1 / 4$, and $t=1$ is the curve for the random flux problem. Since, without an external magnetic field, the band center is at $E=0$ and the localization length is an even function of energy $E$, only the positive energy part is plotted in Fig. 1. From Fig. 1, we can see that both random potential and random flux system have similar general features. There are peaks at $E=1.0$ and the inverse of the localization length increases rapidly as energy approaches $E=3.0$. Peaks at $|E|=1.0$ can be understood from the energy band structure of a pure double-chain system. From Eq. (4), the energy spectrum has two branches which are $E=2 \cos k a \pm 1$ when the interchain coupling is $t=1$. These two branches are centered at $E= \pm 1.0$, respectively. There are two major effects when disorder (random potential or random flux) is added to the system: (i) Translational symmetry is destroyed and states become localized; (ii) Disorder also couples these two branches together. Since $W /\left|E-E_{b}\right|$ (where $E_{b}$ is the energy at the band bottom) is bigger for energies close to band edges for a given disorder strength $W$, one expects that the states near the edges of each branch will be affected more than

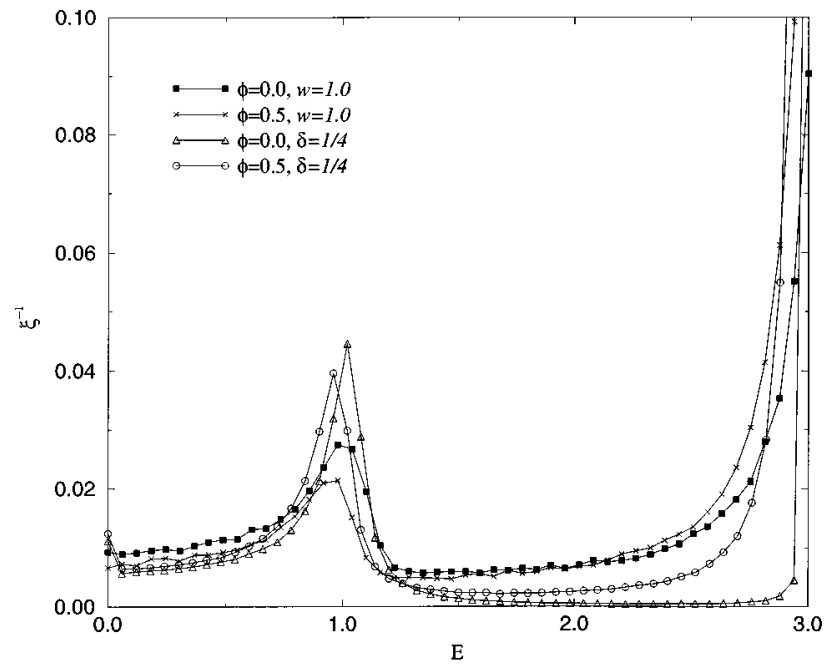

FIG. 1. The inverse of the localization length $\xi^{-1}$ vs energy $E$ for a double-chain system (i) in the presence of random potential $w=1.0$ with uniform magnetic field $\phi=0.0$ and $\phi=0.5(1 / 4 \pi$ quantum flux per plauette), respectively; (ii) in the presence of random magnetic field $\delta=1 / 4$ with uniform magnetic field $\phi=0.0$ and $\phi=0.5$, respectively.

those around the band center. Therefore, the inverse of the localization length is larger at the band center. If this argument is correct, it should work for multiple-chain systems as well. Figure 2 is the numerical results of the inverse of the localization length for a triple-chain random-potential system with $t=1$ and $w=1.0$ and for a triple-chain random flux system with $t=1$ and $\delta=\frac{1}{4}$. It is easy to see that the energy spectrum of the triple-chain systems have three branches with branch edges at $E= \pm(2-\sqrt{2}), \pm 2, \pm(2+\sqrt{2})$. Indeed, there are two peaks in Fig. 2 around these edges as predicted by our argument. When the degree of the randomness is increased, one should expect that all states in each branch will be affected equally strongly, and the peaks at branch edges should diminish accordingly. Figure 3 is the inverse of the localization length vs energy for a double-

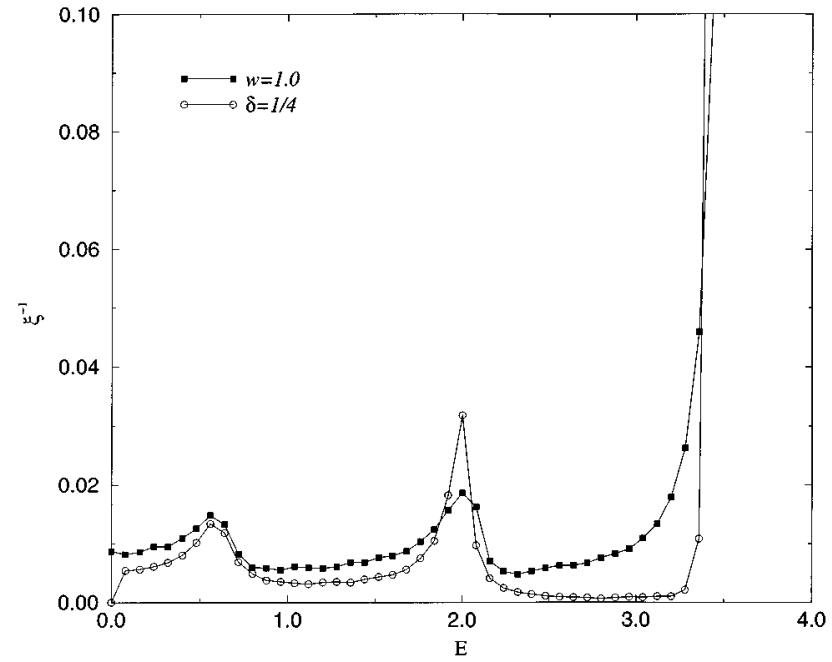

FIG. 2. The inverse of the localization length $\xi^{-1}$ vs energy $E$ for a triple-chain system in the presence of random potential $w=1.0$ and random magnetic fields $\delta=1 / 4$, respectively. 


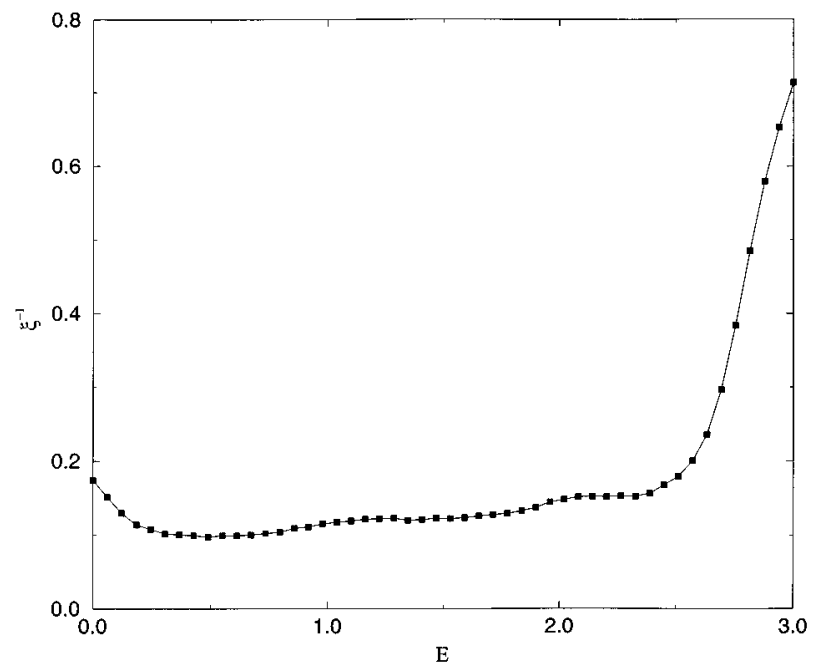

FIG. 3. The inverse of the localization length $\xi^{-1}$ vs energy $E$ for a double-chain strong random-magnetic-field system with $w=0, \delta=1$, and $t=1$.

chain RMF system with $t=1$ and strong randomness $\delta=1$. It is clear that there is no obvious peak in this figure at $E=1$. As the number of chains $M$ increases, the number of branches will increase. Thus, the number of peaks also increases. However, when $M \rightarrow \infty$, the branches edges distribute continuously between $E=[-4,4]$ for $t=1$. Therefore, no peak will appear in a two-dimensional system.

It is interesting to notice that random potential and random flux systems behave differently near the band center. From Fig. 1, random potential system is structureless at the band center. However, random flux system has a peak at the band center $E=0$ while Fig. 2 has a dip at the band center. Numerically, we cannot be sure that the inverse of the localization length at band center $E=0$ is equal to 0 for a triplechain random flux system though there is indication that it is so. Even more interesting, the dip at the band center seems always to exist for an odd-chain RMF system while a peak occurs at the band center for an even-chain RMF system. We do not have a good explanation for the observed even-odd behavior yet.

\section{B. Energy dependence of magnetoproperties of the localization length}

In a random system with the time-reversal symmetry, there is so-called weak localization effect. According to this effect, the coherent backward scattering will be suppressed by a uniform external magnetic field which destroys the time-reversal symmetry. This effect has been used to explain the positive magnetoconductance observed in a dirty metal. A magnetic field, however, has other effects on a wave function, ${ }^{16,13}$ such as changing phases of forward scatterings which may become important on an electron in a state not too close to a band center. In order to see to what extent one can say that the localization length will increase in a weak magnetic field, we studied the energy dependence of the magnetoproperties of the localization length for both random-potential and RMF systems. We find that the localization length can both increase and decrease with a magnetic field for the tight-binding Hamiltonian we have studied.

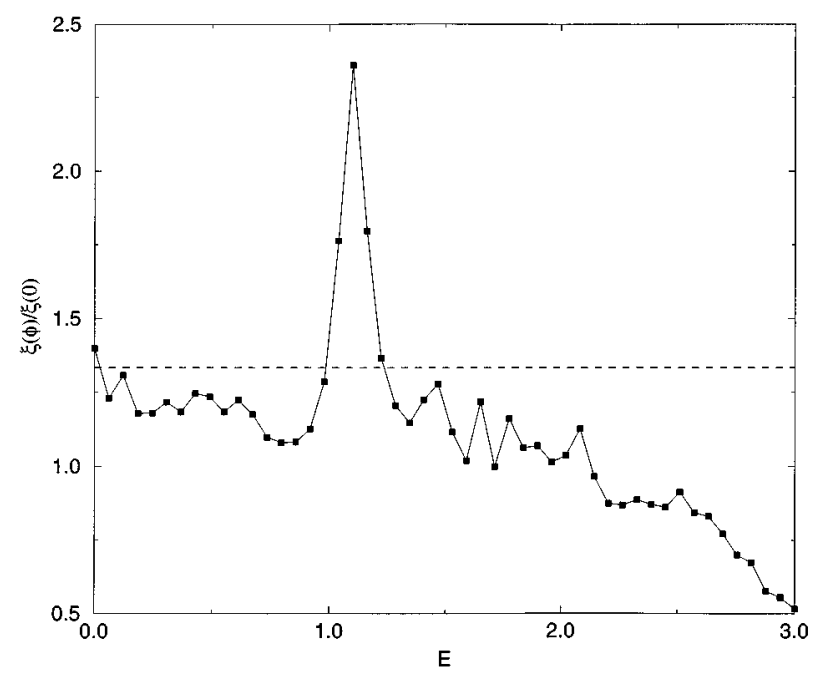

FIG. 4. A plot of $\xi(\phi) / \xi(0)$ vs energy $E$ for a double-chain random-potential system with $w=1.0, \phi=0.5, \delta=0$, and $t=1$.

From Fig. 1, we can see the comparison of the inverse of the localization length for a double-chain random-potential system of $w=1, \delta=0$, and $t=1$ with and without a uniform magnetic field. From Fig. 1, we can see that the magnetoresponse can be divided into two regions. When the electron energy is near the band center, the localization length increases with magnetic field. As the electron energy increases beyond $E=2.0$, the magnetoresponse of the localization length reverses. Therefore, whether the localization length of a given state increases with a magnetic field depends on the energy of the state. This result disagrees clearly with that of weak localization ${ }^{17,10}$ theory, which predicts that for a given energy the localization length $\xi(\phi)$ obeys the relation

$$
\frac{\xi(\phi)}{\xi(0)}=\frac{4}{3},
$$

for a double-chain system. Figure 4 is the plot of $\xi(\phi) / \xi(0)$ vs $E$. Equation (9) holds well for $|E|<1$, but is incorrect outside that energy range. In order to look at the dependence of the localization length on a magnetic field, we plot $\xi(0) / \xi(\phi)$ against $\phi$. Figure 5 is a such graph for various energies in the same system as that in Fig. 1. It is clear that the localization length increases with magnetic field for those states near the band center, and decreases for the states near the band edges.

This magnetoresponse can be explained by the competition of the two effects of magnetic field. The first effect is that the magnetic field destroys the time-reversal symmetry; thus it suppresses the coherent backward scattering. This effect weakens the localization, thus, increases the localization length. The second effect can be understood from Hamiltonian (2) in the pure case. Equation (4) gives the energy band in the presence of a uniform field. According to the formula, the uniform magnetic field shrinks the energy band. It is equivalent to say that a magnetic field tends to push states near band edges to the localized gap states. We will call it the band-edge effect. These two effects compete with each other, and lead to a nontrivial magnetoresponse of the localization length. As the electron energy is closed to the band edge, the band-edge effect dominates the magnetore- 


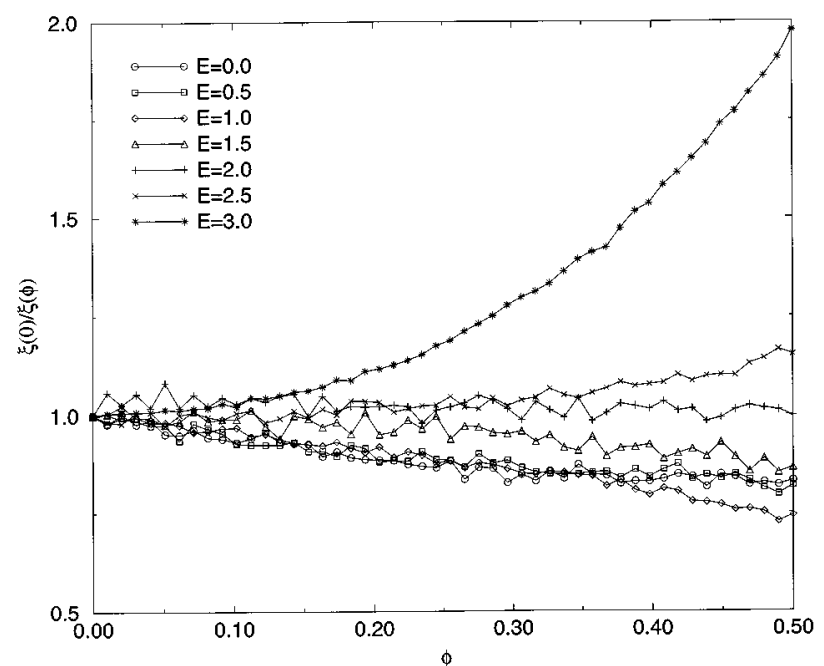

FIG. 5. $\xi(0) / \xi(\phi)$ vs magnetic field $\phi$ for states of various energies $E=0,0.5,1,1.5,2,2.5,3$ in a double-chain randompotential system with $w=1.0, \quad \delta=0$, and $t=1$.

sponse and leads to a decrease in the localization length. On the other hand, for states in the band center, the first effect dominates, and the increase of the localization length is expected. We have to point out that to discuss the magnetoresistance, one has to consider the change of density of states with magnetic field. However, such change can be ignored if weak field is considered. Although Fig. 1 and Fig. 4 showed the results with relative big field $(\phi=0.5)$, we found similar behaviors for the weak-field case, namely, the localization length increases for states near band center and decreases for states near band edge. The same result can also be seen in Fig. 5. Therefore, similar to the localization length, both the negative and positive magnetoresistance are possible in the weak-field limit where the field effect on the density of states can be neglected.

Since there is no time-reversal symmetry in a RMF system, there is no coherent backward scattering. Thus, a weak magnetic field does not have an effect on the backward scat-

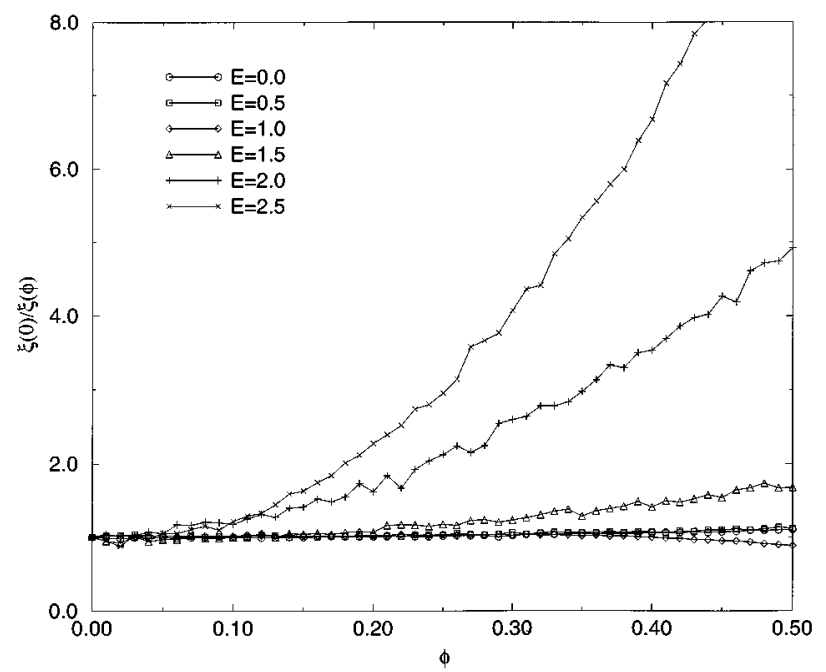

FIG. 6. $\xi(0) / \xi(\phi)$ vs magnetic field $\phi$ for states of various energies $E=0,0.5,1,1.5,2,2.5$ in a double-chain randommagnetic-field system with $w=0, \delta=1 / 4$, and $t=1$.

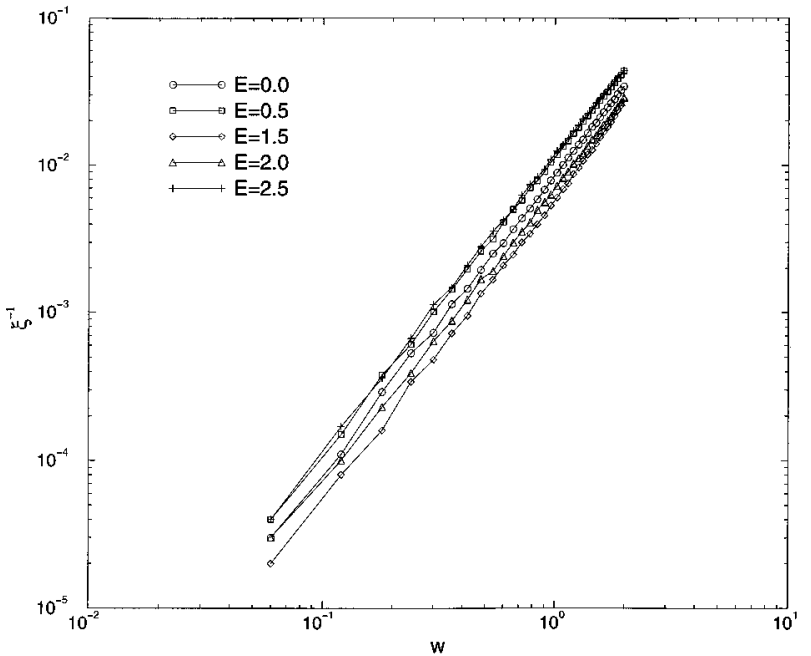

FIG. 7. A log-log plot of the inverse of the localization length vs the randomness $w$ for states of various energies $E=0,0.5,1.5,2$, 2.5 in a double-chain random-potential system with $\delta=0$ and $t=1$.

tering anymore. One should expect that the magnetoresponse of a RMF model is very different from that of randompotential system. We studied a double-chain system in the presence of random magnetic flux. From Fig. 1 (a doublechain random flux system with $w=0, \delta=1 / 4$, and $t=1$ in an external uniform magnetic field $\phi=0$ and $\phi=0.5$, respectively) and Fig. 6, a plot of $\xi(0) / \xi(\phi)$ vs $\phi$ for states of various energies in the same system, we can see that the localization length of a RMF system in general decreases with a uniform external magnetic field except for states in a region of width of $\sim 0.2$ around $E=1$. This feature is consistent with the band-edge effect.

\section{The localization length vs the randomness strength}

It is well known that, in a quasi-one-dimensional randompotential system, the inverse of the localization length $\xi^{-1}$ scales as the square of Anderson randomness $w$ for small

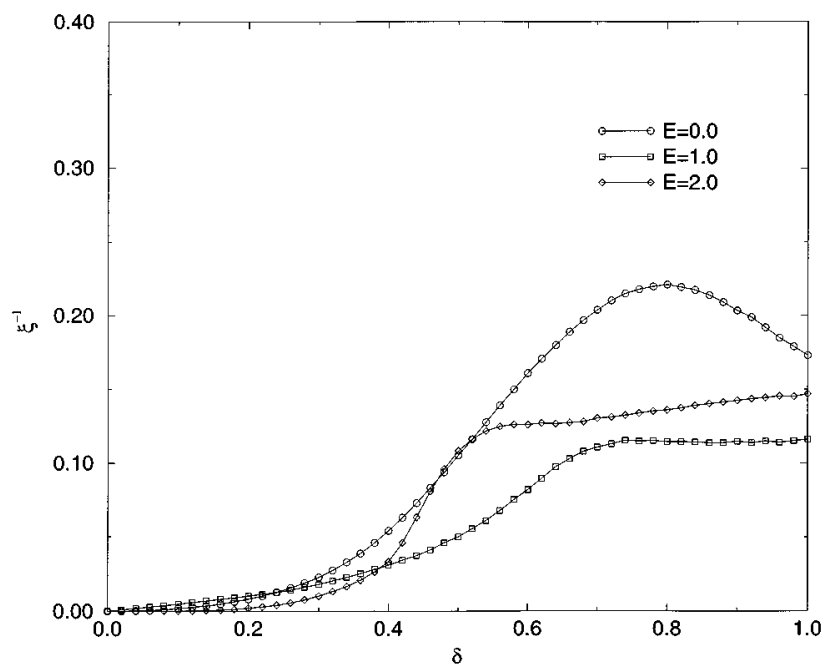

FIG. 8. The inverse of the localization length vs randomness $\delta$ for states of various energies $E=0,1,2$ in a double-chain randommagnetic-field system with $w=0$ and $t=1$. 

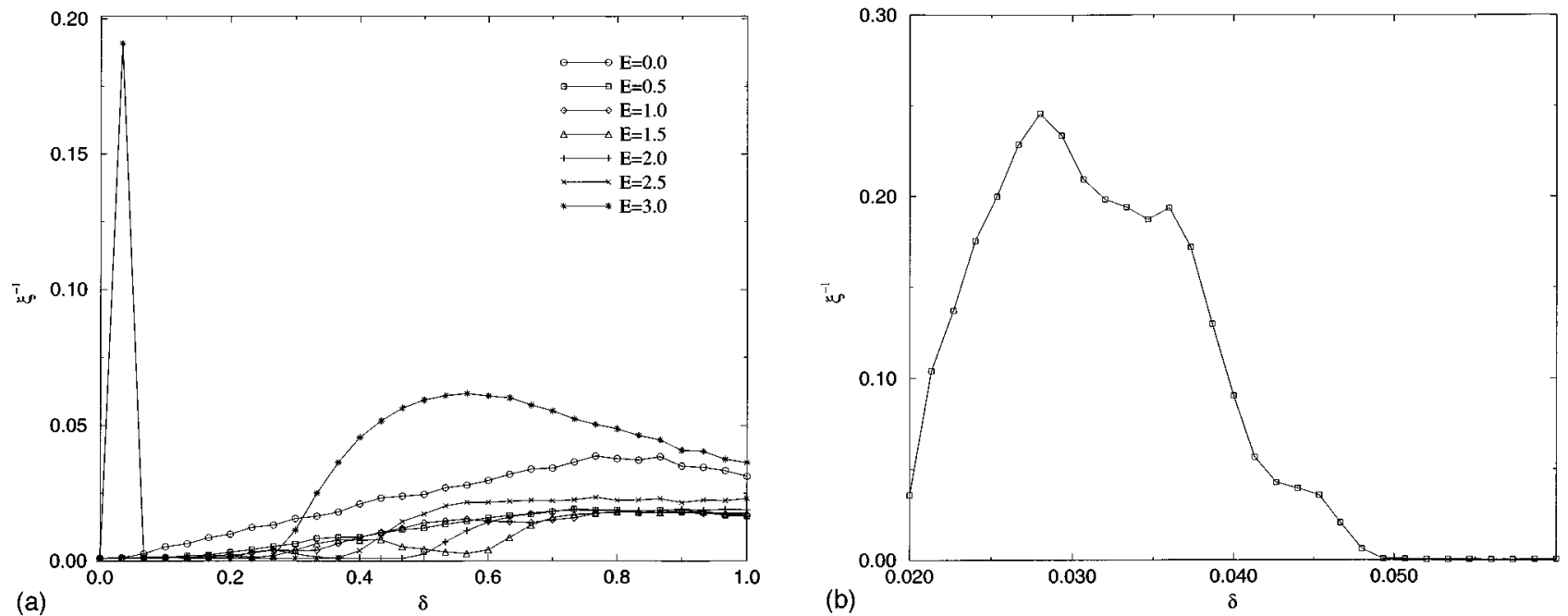

FIG. 9. The inverse of the localization length vs randomness $\delta$ for a 20-chain random-magnetic-field system with $w=0$ and $t=1$. (a) For states of energies $E=0,0.51,1.5,2,2.5,3$. (b) For state $E=3$ around $\delta=0.03$.

$w$. Figure 7 is a log-log plot of the inverse of localization length versus $w$ for a double-chain random-potential system for states of various energies. The randomness dependence of $\xi^{-1}$ can be described well by scaling relation $w^{2}$. For a RMF system, the randomness is on the phases of the hopping coefficients. Because of the periodic condition in the phase, one will expect that the localization length of a given state should saturate when the phase randomness $\delta$ is larger than certain value. Figure 8 shows the inverse of localization length versus the phase randomness for a double-chains RMF system of $t=1$ and for states of energies $E=0,1,2$. Surprisingly, the localization length is not a monotonic function of the randomness $\delta$ in a RMF system. In order to see whether this is a general behavior of a RMF system, we also did calculations on a 20-chain system with $t=1$ and the results are shown in Fig. 9(a). Again, the inverse of localization length is not monotonic in $\delta$, and it saturated at large $\delta$ for some states. Even more surprising, the inverse of the localization length of state $E=3$ has a sharp peak around $\delta=0.03$. The peak height indicates that the localization length is about 5 lattice constants. The detailed studies show that the peak has a finite width and is not a single point as shown in Fig. 9(b). One of the possible explanations of this behavior is that $E=3$ around randomness $\delta=0.03$ is a forbidden state, but it becomes an allowed state for other $\delta$. It is known that the energy spectrum of a two-dimensional lattice in an incommensurate magnetic field is a Cantor set which has a zero measure. ${ }^{18}$ In a disordered system, however, it is difficult to explain why there exists discrete forbidden states in a RMF system. The physical explanation for this peak is still unclear.

\section{ACKNOWLEDGMENTS}

One of the authors (W.L.C.) would like to thank Z.Q. Zhang and T.K. Ng for their useful discussions. This work was supported by UGC, Hong Kong, though RGC, DAG, and RTG grants.
${ }^{1}$ S. C. Zhang and D. P. Arovas, Phys. Rev. Lett. 72, 1886 (1994); V. Kalmeyer and S. C. Zhang, Phys. Rev. B 46, 9889 (1992).

${ }^{2}$ Dongzi Liu and S. D. Sarma, Phys. Rev. B 49, 2677 (1994).

${ }^{3}$ V. Kalmeyer, D. Wei, D. P. Arovas, and S. C. Zhang, Phys. Rev. B 48, 11095 (1993).

${ }^{4}$ T. Sugiyama and N. Nagaosa, Phys. Rev. Lett. 70, 1980 (1993).

${ }^{5}$ Y. Avishai, Y. Hatsugai, and M. Kohmoto, Phys. Rev. B 47, 9561 (1993).

${ }^{6}$ A. G. Aronov, A. D. Mirlin, and P. Wolfle, Phys. Rev. B 49, 16609 (1994); D. V. Khveshchenko and S. V. Meshkov, ibid. 47, 12051 (1993).

${ }^{7}$ D.Z. Liu, X.C. Xie, S. Das Sarma, and S.C. Zhang, Phys. Rev. B 52, 5858 (1995).

${ }^{8}$ B.I. Halperin, P.A. Lee and N. Read, Phys. Rev. B 47, 7312 (1993).

${ }^{9}$ N. Nagaosa and P.A. Lee, Phys. Rev. Lett. 64, 2450 (1990).

${ }^{10}$ Y. Avishai and J. Bar-Touv, Phys. Rev. B 51, 8069 (1995).
${ }^{11}$ C. Pryor, A. Zee, Phys. Rev. B 46, 1 (1992).

${ }^{12}$ A. MacKinnon and B. Kramer, Z. Phys B 53, 1 (1983).

${ }^{13}$ E. Medina, M. Kardar, Y. Shapir, and X. R. Wang, Phys. Rev. Lett. 64, 1816 (1990); X. R. Wang, Y. Shapir, E. Medina, and M. Kardar, Phys. Rev. B 42, 4559 (1990); Y. Shapir and X. R. Wang, Mod. Phys. Lett. B 4, 1301 (1990).

${ }^{14}$ W. L. Chan, Localization and Magneto-Properties in Quasi-OneDimensional Disordered Systems, Master of Philosophy thesis, The Hong Kong University of Science \& Technology, 1995.

${ }^{15}$ A. Crisanti, G. Paladin, and A. Vulpiani, Product of Random Matrices (Springer-Verlag, Berlin, 1993).

${ }^{16}$ X. R. Wang, Phys. Rev. B 51, 9310 (1995); X. R. Wang, Phys. Rev. B 53, 12035 (1996).

${ }^{17}$ J. L. Pichard, M. Sanquer, K. Slevin, and P. Derbay, Phys. Rev. Lett. 65, 1812 (1990)

${ }^{18}$ D. R. Hofstadter, Phys. Rev. B 14, 2239 (1976). 